ECM-UB-PF 08/22

December 2008

arXiv:0812.1114 [hep-ph]

\title{
Effective description of squark interactions
}

\author{
Jaume Guasch $^{a, b}$, SiAnnah Peñaranda ${ }^{c}$, RaÜl SÁnchez-Florit $^{d, b}$ \\ ${ }^{a}$ Departament de Física Fonamental, \\ Universitat de Barcelona, Diagonal 647, E-08028 Barcelona, Catalonia, Spain \\ ${ }^{b}$ Institut de Ciències del Cosmos de la Universitat de Barcelona, \\ Diagonal 647, E-08028 Barcelona, Catalonia, Spain \\ ${ }^{c}$ Departamento de Física Teórica, Facultad de Ciencias, \\ Universidad de Zaragoza, E-50009 Zaragoza, Spain \\ d Departament d'Estructura i Constituents de la Matèria, \\ Universitat de Barcelona, Diagonal 647, E-08028 Barcelona, Catalonia, Spain \\ E-mails: jaume.guasch@ub.edu,siannah@unizar.es, florit@ffn.ub.es
}

\begin{abstract}
We propose an effective description of squark interactions with charginos/neutralinos. We recompute the strong corrections to squark partial decay widths, and compare the full one-loop computation with the effective description. The effective description includes the effective Yukawa couplings, and another logarithmic term which encodes the supersymmetry-breaking. The proposed effective couplings reproduce correctly the radiative-corrected partial decay widths of the squark decays into charginos and neutralinos in all relevant regions of the parameter space.
\end{abstract}




\section{Introduction}

The Standard Model (SM) of the strong and electroweak interactions is the present paradigm of particle physics. Its validity has been tested to a level better than one per mille at particle accelerators [1]. Nevertheless, there are arguments against the SM being the fundamental model of particle interactions [2], giving rise to the investigation of competing alternative or extended models, which can be tested at high-energy colliders, such as the Large Hadron Collider (LHC) [3,4], or a $500-1000 \mathrm{GeV}, e^{+} e^{-}$International Linear Collider (ILC) [5,6]. One of the most promising possibilities for physics beyond the SM is the incorporation of Supersymmetry (SUSY), which leads to a renormalizable field theory with precisely calculable predictions to be tested in present and future experiments. The simplest supersymmetric extension of the SM is the Minimal Supersymmetric Standard Model (MSSM) [7-10]. Among the most important phenomenological consequences of SUSY models, is the prediction of new particles. There is much excitement for the possibility of discovering these new particles at the recently built LHC [11,12], and their properties will need to be precisely measured to confirm (or refute) that they belong to a SUSY model. This last effort might be better suited for the ILC [5, 6, 13-15], currently being projected. This job needs the performance of precision measurements, but also of precision computations which are well suited for experimental comparisons. In the present work we will focus on the properties of the SUSY partners of the SM quarks - the squarks.

Once produced, squarks will decay in a way dependent on the model parameters (see e.g. [16]). If gluinos (the fermionic SUSY partners of gluons) are light enough, squarks will mainly decay into gluinos and quarks $(\tilde{q} \rightarrow q \tilde{g})[17,18]$, which proceeds trough a coupling constant of strong strength. If the mass difference among different squarks is large enough, some squarks can decay via a bosonic channel into an electroweak gauge boson and another squark $\left(\tilde{q}_{a} \rightarrow \tilde{q}_{b}^{\prime}\left(Z, W^{ \pm}\right)\right)$, and if Higgs bosons are light enough, also the scalar decay channels are available $\left(\tilde{q}_{a} \rightarrow \tilde{q}_{b}^{\prime}\left(h^{0}, H^{0}, A^{0}, H^{ \pm}\right)\right)$[19-22], which can be dominant for third generation squarks due to the large Yukawa couplings. Otherwise, the main decay channels of squarks are their partial decays into charginos/neutralinos (the fermionic SUSY partners of the electroweak gauge and Higgs bosons) and quarks $\left(\tilde{q} \rightarrow q^{\prime} \chi\right)$. Some of those channels are expected to be always open, given the large mass difference between quarks and squarks, and that the charginos/neutralinos are expected to be lighter than most of squarks in the majority of SUSY-breaking models. In the few cases in which these channels are closed, the squarks will decay through flavour changing neutral channels [23-25], or through three- or four-body decay channels involving a non-resonant SUSY particle [26-31].

Here we will concentrate on the squark decay channels involving charginos and neutralinos. Their partial decay widths were computed some time ago, including the radiative corrections due to the strong (QCD) [32-34], and the electroweak (EW) [35-38] sectors of the theory. These radiative corrections are large in certain regions of the parameter space [37], and their complicated expressions are not suitable for their introduction in the monte-carlo programs used for experimental analyses. In this work we present approximations for the partial decay widths of squarks into charginos and neutralinos, including 
the QCD corrections, and compare these approximations against the fixed-order one-loop corrected partial widths.

In section 2 we introduce our notation and conventions for particles and couplings, and set up the numerical values that we will use in our analysis, section 3 presents the QCD one-loop computation of the partial decay widths and shows some numerical examples, in section 4 we perform a renormalization group analysis of the partial decay widths, in section 5 we perform a numerical comparison of the one-loop and renormalization group computations, and finally section 6 shows our conclusions.

\section{Notation, conventions and numerical setup}

To describe the computation of the partial decay widths, we will follow the conventions of Ref. [39]. Throughout this work we will use a third-generation notation to describe quarks and squarks, but the analytic results and conclusions are completely general, and can be used for quarks-squarks of any generation. We will show numerical results only for third generation quarks/squarks (top $t$ /stop $\tilde{t}$ /bottom $b$ /sbottom $\tilde{b}$ ), since their decay widths are the ones that present the most interesting properties.

We will study the partial decay widths of sfermions into fermions and charginos/neutralinos,

$$
\Gamma\left(\tilde{f} \rightarrow f^{\prime} \chi\right)
$$

We denote the two sfermion-mass eigenvalues by $m_{\tilde{f}_{a}}(a=1,2)$, with $m_{\tilde{f}_{1}}<m_{\tilde{f}_{2}}$. The sfermion-mixing angle $\theta_{f}$ is defined by the transformation relating the weak-interaction $\left(\tilde{f}_{a}^{\prime}=\tilde{f}_{L}, \tilde{f}_{R}\right)$ and the mass eigenstate $\left(\tilde{f}_{a}=\tilde{f}_{1}, \tilde{f}_{2}\right)$ sfermion bases:

$$
\tilde{f}_{a}=R_{a b}^{(f)} \tilde{f}_{b}^{\prime} ; \quad R^{(f)}=\left(\begin{array}{cc}
\cos \theta_{f} & -\sin \theta_{f} \\
\sin \theta_{f} & \cos \theta_{f}
\end{array}\right)
$$

By this basis transformation, the sfermion mass matrix,

$$
\mathcal{M}_{\tilde{f}}^{2}=\left(\begin{array}{cc}
M_{\tilde{f}_{\mathrm{L}}}^{2}+m_{f}^{2}+c_{2 \beta}\left(T_{3}-Q s_{W}^{2}\right) M_{Z}^{2} & m_{f} M_{f}^{L R} \\
m_{f} M_{f}^{L R} & M_{\tilde{f}_{\mathrm{R}}}^{2}+m_{f}^{2}+Q c_{2 \beta} s_{W}^{2} M_{Z}^{2}
\end{array}\right),
$$

becomes diagonal: $R^{(f)} \mathcal{M}_{\tilde{f}}^{2} R^{(f) \dagger}=\operatorname{diag}\left\{m_{\tilde{f}_{1}}^{2}, m_{\tilde{f}_{2}}^{2}\right\} . M_{\tilde{f}_{\mathrm{L}}}^{2}$ is the soft-SUSY-breaking mass parameter of the $S U(2)_{L}$ doublet 1 , whereas $M_{\tilde{f}_{\mathrm{R}}}^{2}$ is the soft-SUSY-breaking mass parameter of the singlet. $T_{3}$ and $Q$ are the usual third component of the isospin and the electric charge respectively, $m_{f}$ is the corresponding fermion mass, and $s_{W}$ is the sinus of the weak mixing angle.2 The mixing parameters in the non-diagonal entries read

$$
M_{b}^{L R}=A_{b}-\mu \tan \beta \quad, \quad M_{t}^{L R}=A_{t}-\mu / \tan \beta .
$$

\footnotetext{
${ }^{1}$ With $M_{\tilde{t}_{L}}=M_{\tilde{b}_{L}}$ due to $S U(2)_{L}$ gauge invariance.

${ }^{2}$ We abbreviate trigonometric functions by their initials, like $s_{W} \equiv \sin \theta_{W}, c_{2 \beta} \equiv \cos (2 \beta), t_{W} \equiv s_{W} / c_{W}$, etc.
} 
$A_{b, t}$ are the trilinear soft-SUSY-breaking couplings, $\mu$ is the higgsino mass parameter, and $\tan \beta$ is the ratio between the vacuum expectation values of the two Higgs doublets $\tan \beta=v_{2} / v_{1}$. The input parameters in the sfermion sector are then:

$$
\left(M_{\tilde{f}_{\mathrm{L}}}, M_{\tilde{b}_{R}}, M_{\tilde{t}_{R}}, A_{b}, A_{t}, \mu, \tan \beta\right),
$$

for each sfermion doublet. From them, we can derive the masses and mixing angles:

$$
\left(m_{\tilde{b}_{1}}, m_{\tilde{b}_{2}}, \theta_{b}\right),\left(m_{\tilde{t}_{1}}, m_{\tilde{t}_{2}}, \theta_{t}\right)
$$

For the trilinear couplings, we require the approximate (necessary) condition

$$
A_{q}^{2}<3\left(m_{\tilde{t}}^{2}+m_{\tilde{b}}^{2}+M_{H}^{2}+\mu^{2}\right),
$$

where $m_{\tilde{q}}$ is of the order of the average squark masses for $\tilde{q}=\tilde{t}, \tilde{b}$, to avoid colour-breaking minima in the MSSM Higgs potential [40-43].

Although the tree-level chargino $\left(\chi^{+}\right)$-neutralino $\left(\chi^{0}\right)$ sector is well known, we give here a short description, in order to set our conventions. We start by constructing the following set of Weyl spinors:

$$
\begin{aligned}
& \Gamma^{+} \equiv\left(-i \tilde{W}^{+}, \tilde{H}_{2}^{+}\right) \\
& \Gamma^{-} \equiv\left(-i \tilde{W}^{-}, \tilde{H}_{1}^{-}\right) \\
& \Gamma^{0} \equiv\left(-i \tilde{B}^{0},-i \tilde{W}_{3}^{0}, \tilde{H}_{1}^{0}, \tilde{H}_{2}^{0}\right) .
\end{aligned}
$$

The mass Lagrangian in this basis reads

$$
\mathcal{L}_{M}=-\frac{1}{2}\left(\Gamma^{+}, \Gamma^{-}\right)\left(\begin{array}{cc}
0 & \mathcal{M}^{T} \\
\mathcal{M} & 0
\end{array}\right)\left(\begin{array}{l}
\Gamma^{+} \\
\Gamma^{-}
\end{array}\right)-\frac{1}{2}\left(\Gamma_{1}, \Gamma_{2}, \Gamma_{3}, \Gamma_{4}\right) \mathcal{M}^{0}\left(\begin{array}{l}
\Gamma_{1} \\
\Gamma_{2} \\
\Gamma_{3} \\
\Gamma_{4}
\end{array}\right)+\text { h.c. }
$$

where we have defined

$$
\begin{aligned}
\mathcal{M}= & \left(\begin{array}{ccc}
M & \sqrt{2} M_{W} s_{\beta} \\
\sqrt{2} M_{W} c_{\beta} & \mu
\end{array}\right), \\
\mathcal{M}^{0}= & \left(\begin{array}{cccc}
M^{\prime} & 0 & M_{Z} c_{\beta} s_{W} & -M_{Z} s_{\beta} s_{W} \\
0 & M & -M_{Z} c_{\beta} c_{W} & M_{Z} s_{\beta} c_{W} \\
M_{Z} c_{\beta} s_{W} & -M_{Z} c_{\beta} c_{W} & 0 & -\mu \\
-M_{Z} s_{\beta} s_{W} & M_{Z} s_{\beta} c_{W} & -\mu & 0
\end{array}\right),
\end{aligned}
$$

with $M$ and $M^{\prime}$ the $S U(2)_{L}$ and $U(1)_{Y}$ soft-SUSY-breaking gaugino masses. The fourcomponent mass-eigenstate fields are related to the ones in (17) by

$$
\chi_{i}^{+}=\left(\begin{array}{c}
V_{i j} \Gamma_{j}^{+} \\
U_{i j}^{*} \bar{\Gamma}_{j}^{-}
\end{array}\right) \quad, \quad \chi_{i}^{-}=\mathcal{C} \bar{\chi}_{i}^{+T}=\left(\begin{array}{c}
U_{i j} \Gamma_{j}^{-} \\
V_{i j}^{*} \bar{\Gamma}_{j}^{+}
\end{array}\right) \quad, \quad \chi_{\alpha}^{0}=\left(\begin{array}{c}
N_{\alpha \beta} \Gamma_{\beta}^{0} \\
N_{\alpha \beta}^{*} \bar{\Gamma}_{\beta}^{0}
\end{array}\right)=\mathcal{C} \bar{\chi}_{\alpha}^{0 T}
$$


where $U, V$ and $N$ are in general complex matrices that diagonalize the mass-matrices (9):

$$
\begin{aligned}
& U^{*} \mathcal{M} V^{\dagger}=\mathcal{M}_{D}=\operatorname{diag}\left(M_{1}, M_{2}\right)\left(0<M_{1}<M_{2}\right), \\
& N^{*} \mathcal{M}^{0} N^{\dagger}=\mathcal{M}_{D}^{0}=\operatorname{diag}\left(M_{1}^{0}, M_{2}^{0}, M_{3}^{0}, M_{4}^{0}\right)\left(0<M_{1}^{0}<M_{2}^{0}<M_{3}^{0}<M_{4}^{0}\right) .
\end{aligned}
$$

Using this notation, the tree-level interaction Lagrangian between fermion-sfermion(chargino or neutralino) reads [37]

$$
\begin{aligned}
\mathcal{L}_{\chi \tilde{f} f^{\prime}} & =\sum_{a=1,2} \sum_{r} \mathcal{L}_{\chi_{r} \tilde{f}_{a} f^{\prime}}+\text { h.c. }, \\
\mathcal{L}_{\chi_{r} \tilde{f}_{a} f^{\prime}} & =-g \tilde{f}_{a}^{*} \bar{\chi}_{r}\left(A_{+a r}^{(f)} P_{L}+A_{-a r}^{(f)} P_{R}\right) f^{\prime} .
\end{aligned}
$$

Here we have adopted a compact notation, where $f^{\prime}$ is either $f$ or its $S U(2)_{L}$ partner for $\chi_{r}$ being a neutralino or a chargino, respectively. Roman characters $a, b \ldots$ are reserved for sfermion indices and $i, j, \ldots$ for chargino indices; Greek indices $\alpha, \beta, \ldots$ denote neutralinos; Roman indices $r, s \ldots$ indicate either a chargino or a neutralino. For example, the topsquark interactions with charginos are obtained by replacing $f \rightarrow t, f^{\prime} \rightarrow b, \chi_{r} \rightarrow \chi_{r}^{-}$, $r=1,2$. The coupling matrices that encode the dynamics are given by

$$
\begin{aligned}
A_{+a i}^{(t)} & =R_{a 1}^{(t)} V_{i 1}^{*}-\lambda_{t} R_{a 2}^{(t)} V_{i 2}^{*}, \\
A_{-a i}^{(t)} & =-\lambda_{b} R_{a 1}^{(t)} U_{i 2} \\
A_{+a \alpha}^{(t)} & =\frac{1}{\sqrt{2}}\left(R_{a 1}^{(t)}\left(N_{\alpha 2}^{*}+Y_{L} t_{W} N_{\alpha 1}^{*}\right)+\sqrt{2} \lambda_{t} R_{a 2}^{(t)} N_{\alpha 4}^{*}\right), \\
A_{-a \alpha}^{(t)} & =\frac{1}{\sqrt{2}}\left(\sqrt{2} \lambda_{t} R_{a 1}^{(t)} N_{\alpha 4}-Y_{R}^{t} t_{W} R_{a 2}^{(t)} N_{\alpha 1}\right), \\
A_{+a i}^{(b)} & =R_{a 1}^{(b)} U_{i 1}^{*}-\lambda_{b} R_{a 2}^{(b)} U_{i 2}^{*} \\
A_{-a i}^{(b)} & =-\lambda_{t} R_{a 1}^{(b)} V_{i 2} \\
A_{+a \alpha}^{(b)} & =-\frac{1}{\sqrt{2}}\left(R_{a 1}^{(b)}\left(N_{\alpha 2}^{*}-Y_{L} t_{W} N_{\alpha 1}^{*}\right)-\sqrt{2} \lambda_{b} R_{a 2}^{(b)} N_{\alpha 3}^{*}\right), \\
A_{-a \alpha}^{(b)} & =-\frac{1}{\sqrt{2}}\left(-\sqrt{2} \lambda_{b} R_{a 1}^{(b)} N_{\alpha 3}+Y_{R}^{b} t_{W} R_{a 2}^{(b)} N_{\alpha 1}\right),
\end{aligned}
$$

with $Y_{L}$ and $Y_{R}^{t, b}$ the weak hypercharges of the left-handed $S U(2)_{L}$ doublet and righthanded singlet fermion, and $\lambda_{t}=m_{t} /\left(\sqrt{2} M_{W} \sin \beta\right)$ and $\lambda_{b}=m_{b} /\left(\sqrt{2} M_{W} \cos \beta\right)$ are the Yukawa couplings normalized to the $S U(2)_{L}$ gauge coupling constant $g$. Note the following, each coupling is formed by two parts: the gaugino part, formed exclusively by gauge couplings, and the higgsino part, which contains factors of the quark masses, each of these parts will receive different kinds of corrections (see below).

Using these definitions, the tree-level partial decay widths read

$$
\begin{aligned}
\Gamma_{a r}^{\text {tree }}= & \Gamma^{\text {tree }}\left(\tilde{f}_{a} \rightarrow f^{\prime} \chi_{r}\right)=\frac{g^{2}}{16 \pi m_{\tilde{f}_{a}}^{3}} \lambda\left(m_{\tilde{f}_{a}}^{2}, M_{r}^{2}, m_{f^{\prime}}^{2}\right) \times \\
& \times\left[\left(m_{\tilde{f}_{a}}^{2}-M_{r}^{2}-m_{f^{\prime}}^{2}\right)\left(\left|A_{+a r}^{(f)}\right|^{2}+\left|A_{-a r}^{(f)}\right|^{2}\right)-4 m_{f^{\prime}} M_{r} \operatorname{Re}\left(A_{+a r}^{(f)} A_{-a r}^{(f) *}\right)\right]
\end{aligned}
$$


with $\lambda\left(x^{2}, y^{2}, z^{2}\right)=\sqrt{\left[x^{2}-(y-z)^{2}\right]\left[x^{2}-(y+z)^{2}\right]}$.

\section{$2.1 \quad$ Numerical setup}

For the numerical analysis and plots we will use fixed values for the SUSY parameters, and make plots by changing one parameter at a time. For the central values of the parameters we take:

$$
\begin{gathered}
\tan \beta=5, \mu=300 \mathrm{GeV}, M=200 \mathrm{GeV}, M_{\tilde{f}_{\mathrm{L}}}=800 \mathrm{GeV}, m_{\tilde{g}}=3000 \mathrm{GeV}, \\
M_{\mathrm{SUSY}} \equiv M_{\tilde{f}_{\mathrm{R}}}=1000 \mathrm{GeV}, A_{t}=A_{b}=2 M_{\tilde{f}_{\mathrm{L}}}+\mu / \tan \beta=1660 \mathrm{GeV},
\end{gathered}
$$

where we have introduced a parameter $M_{\mathrm{SUSY}}$ as a shortcut for all the SUSY mass parameters which are not explicitly given. We use the GUT relation $M^{\prime}=5 / 3 t_{W}^{2} M$ for the bino mass parameter. For the SM parameters we use $m_{t}=171.2 \mathrm{GeV}, m_{b}=4.7 \mathrm{GeV}$, $\alpha_{s}\left(M_{Z}\right)=0.1172, s_{W}^{2}=0.221, M_{Z}=91.1875 \mathrm{GeV}, 1 / \alpha=137.035989$. The renormalization scale $Q$ is taken to be the physical mass of the decaying squark. The value of the trilinear couplings $A_{b, t}$ is given by the algebraic expression, the given numerical value corresponds to the default values of the other parameters, this numerical value will change in the plots, the chosen expression allows to show plots with a significant parameter variation avoiding colour-breaking-vacuum conditions (6). With these input parameters, the central values for the physical SUSY particle masses are:

$$
\begin{aligned}
M_{\chi^{+}} & =(170.40,337.50) \mathrm{GeV}, \\
M_{\chi^{0}} & =(89.52,172.28,305.46,338.58) \mathrm{GeV}, \\
m_{\tilde{b}} & =(802.05,1000.30) \mathrm{GeV}, \\
m_{\tilde{t}} & =(720.55,1084.25) \mathrm{GeV} .
\end{aligned}
$$

It is illustrative to identify the largest EW-basis component in each physical state. Of course, we have performed our computation using the full numerical mixing among the EW-basis and the physical-basis components, but this identification will help us to analyze the numerical results. The lightest squarks $\left(\tilde{t}_{1}, \tilde{b}_{1}\right)$ are predominantly left-handed, the lightest chargino and neutralinos $\left(\chi_{1}^{+}, \chi_{1}^{0}, \chi_{2}^{0}\right)$ are predominantly gaugino-like, whereas the heaviest ones $\left(\chi_{2}^{+}, \chi_{3}^{0}, \chi_{4}^{0}\right)$ are predominantly higgsino-like. Of course, the parameters in eq. (14) are just and example for illustrative purposes, we have checked that our conclusions hold for a wide range of the parameter space.

\section{QCD Corrections}

Following this setup, we have computed the full one-loop QCD corrections to the squark partial decay widths into charginos and neutralinos (1). The renormalization prescriptions follow that of Ref. [37]. The QCD corrections include contributions from gluon loops, gluino loops, and gluon bremsstrahlung. The full one-loop corrections have been performed using the FeynArts/FormCalc/LoopTools packages [39,44-48]. We have used dimensional 
reduction for the regularization of ultraviolet (UV) divergences, and a small gluon mass to regularize the infrared (IR) divergences. The three-body phase-space integration of the real gluon emission is performed analytically over the full energy range, and the dependence on the gluon mass is seen to cancel between the virtual and the real corrections. We have found full agreement with previous works [33,34,37,38], and will not repeat the full lengthy formulae here. The corrections are seen to be numerically large, specially in certain regions of the parameter space [37], specially those involving processes with a bottom-squark in the initial state, and in a regime of large $\tan \beta$ values.

We follow the hints from Higgs-boson physics [49-52], and define effective Yukawa couplings which should encode the leading part of the corrections [50]:

$$
\begin{aligned}
\lambda_{b}^{e f f .} & \equiv \frac{m_{b}^{e f f .}}{v_{1}} \equiv \frac{m_{b}(Q)}{v_{1}\left(1+\Delta m_{b}\right)}, \\
\lambda_{t}^{e f f .} & \equiv \frac{m_{t}^{e f f .}}{v_{2}} \equiv \frac{m_{t}(Q)}{v_{2}\left(1+\Delta m_{t}\right)}
\end{aligned}
$$

where $m_{q}(Q)$ is the running quark mass and $\Delta m_{q}$ is the finite threshold correction. The SUSY-QCD contributions to $\Delta m_{q}$ are:

$$
\begin{aligned}
\Delta m_{b}^{S Q C D} & =\frac{2 \alpha_{s}}{3 \pi} m_{\tilde{g}} \mu \tan \beta I\left(m_{\tilde{b_{1}}}, m_{\tilde{b_{2}}}, m_{\tilde{g}}\right), \\
\Delta m_{t}^{S Q C D} & =\frac{2 \alpha_{s}}{3 \pi} m_{\tilde{g}} \frac{\mu}{\tan \beta} I\left(m_{\tilde{t_{1}}}, m_{\tilde{t_{2}}}, m_{\tilde{g}}\right),
\end{aligned}
$$

where the function $I(a, b, c)$ is the scalar three-point function at zero momentum transfer, and reads:

$$
I(a, b, c)=\frac{a^{2} b^{2} \ln \left(a^{2} / b^{2}\right)+b^{2} c^{2} \ln \left(b^{2} / c^{2}\right)+c^{2} a^{2} \ln \left(c^{2} / a^{2}\right)}{\left(a^{2}-b^{2}\right)\left(b^{2}-c^{2}\right)\left(a^{2}-c^{2}\right)} .
$$

The effective description of squark decays consists in replacing the tree-level quark masses in the couplings (12) by the effective Yukawa couplings of eq. (16), and use this lagrangian to compute the partial decay width, schematically:

$$
\Gamma^{Y u k .-e f f .}=\Gamma^{\text {tree }}\left(m_{q}^{e f f \cdot}\right)
$$

This expression contains the large one-loop corrections from the finite threshold corrections (16), but it also contains higher order corrections. At this point we can make the following: make a computation that combines the higher order effects (which ignore the effects of external momenta) and the fixed one-loop (which ignore the higher order effects). At the same time, this will allow us to quantify the degree of accuracy obtained by the effective description. We define a Yukawa-improved decay width computation:

$$
\Gamma^{Y u k .-i m p .} \equiv \Gamma^{\text {tree }}\left(m_{q}^{\text {eff. }}\right)+\left(\Gamma^{1-l o o p}-\Gamma^{1-l o o p Y u k .-e f f \cdot}\right) \equiv \Gamma^{\text {tree }}\left(m_{q}^{\text {eff. }}\right)\left(1+\delta^{Y u k .-r e m .}\right)
$$


where

$$
\begin{aligned}
\Gamma^{1-\text { loop }} & =\Gamma^{\text {tree }}+\delta \Gamma^{1-\text { loop }} \\
\delta^{1-\text { loop }} & =\frac{\delta \Gamma^{1-\text { loop }}}{\Gamma^{\text {tree }}} \\
\delta^{\text {Yuk.-rem. }} & =\frac{\Gamma^{1-\text { loop }}-\Gamma^{1-\text { loop Yuk.-eff. }}}{\Gamma^{\text {tree }}\left(m_{q}^{\text {eff. }}\right)}
\end{aligned}
$$

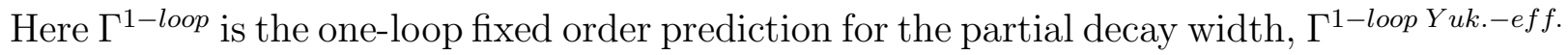
is the one-loop expansion of the prediction using effective couplings, and therefore, the remainder contribution $\left(\delta^{Y u k .-r e m .}\right)$ is the part of the one-loop contribution that can not be described by the Yukawa effective couplings, it quantifies the approximation done by the effective description.

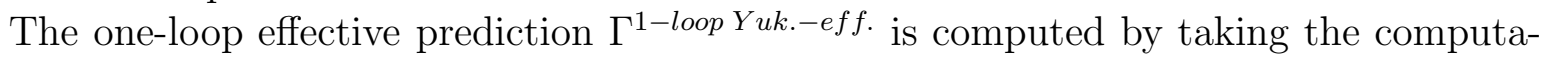
tion using effective couplings (18), expanding it in series, and keeping only the one-loop terms. Specifically:

$$
m(Q)=m(m)\left[1-\frac{2}{\pi} \alpha_{s}(Q) \log \left(\frac{Q}{m}\right)+\ldots\right]
$$

and

$$
m^{e f f .}=m(Q)(1-\Delta m),
$$

therefore, the part of the one-loop effective mass is:

$$
\delta m^{e f f .}=m(m)\left[-\frac{2}{\pi} \alpha_{s}(Q) \log \left(\frac{Q}{m}\right)-\Delta m\right]
$$

this is the mass that will be used in the effective Yukawa couplings to compute $\Gamma^{1-l o o p ~ Y u k .-e f f}$, and $m(m)$ is the running quark mass at the quark mass scale. Finally, we define a Yukawaimproved correction factor in the following way:

$$
\delta^{Y u k .-i m p .}=\frac{\Gamma^{Y u k .-i m p .}-\Gamma^{\text {tree }}\left(m_{q}\right)}{\Gamma^{\text {tree }}\left(m_{q}\right)} .
$$

All these definitions will allow us to precisely analyze the approximations. As an example, Fig. 1 shows the partial decay width (and the relative correction) of a topsquark decaying into the lightest chargino, as a function of the $S U(2)_{L}$ squark mass scale $M_{\tilde{f}_{\mathrm{L}}}$ (3) , the rest of the parameters are given in (14). We see a big dip in the corrections for squark masses around $1250 \mathrm{GeV}$, with negative corrections surpassing $-100 \%$ - which would mean a negative decay width, which obviously does not make sense. What happens is that, for this very special setup of parameters, the tree-level computation of the partial decay width vanishes, so the one-loop contribution exceeds the tree-level prediction. Under these circumstances one-loop perturbation theory does not hold, and we can not claim the validity of any result obtained by the one-loop perturbative expansion, that is: we can not 


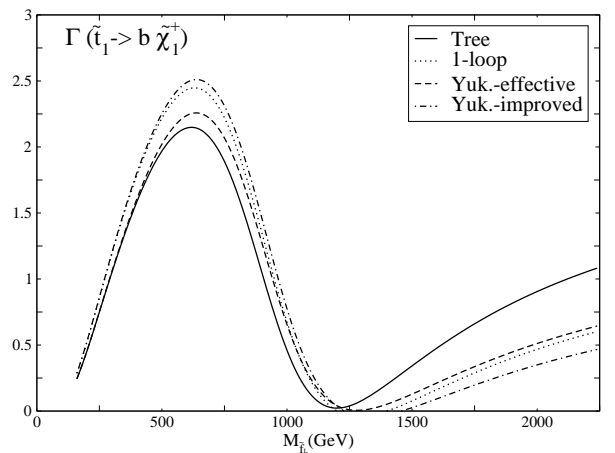

(a)

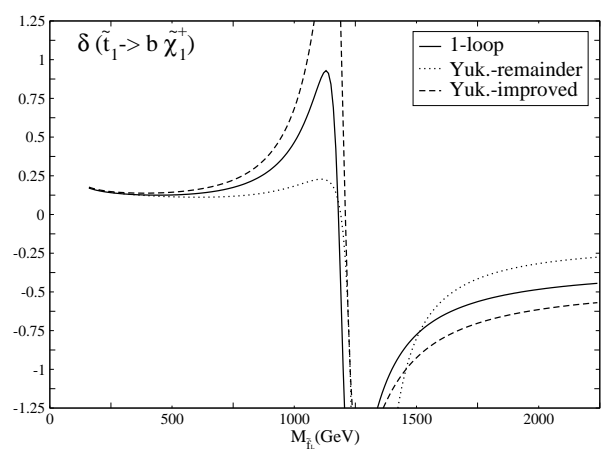

(b)

Figure 1: a) Partial decay width and b) relative corrections to the top-squark decay width into the lightest chargino as a function of the $S U(2)_{L}$ squark mass scale $M_{\tilde{f}_{\mathrm{L}}}$, for the parameters of eq. (14).

give a prediction for the decay width in those parts of the parameter space in the present approximation. Note, also, that the effective prediction $\Gamma^{Y u k .-e f f}$. (18) is (by definition) a positive quantity, therefore the effective description can not reproduce the one-loop result at all, which means a large remainder $\delta^{Y u k .-r e m}$. (20). However, there are a couple of circumstances surrounding these situations: first of all, they appear in tiny regions of the parameter space; second, and more important, this effect occurs precisely on decay channels that have a negligibly small branching ratio, and therefore are phenomenologically irrelevant. We can see that the dip in Fig. 1 $1 \mathrm{~b}$ around $1250 \mathrm{GeV}$ coincides with the minimum of the partial decay width in Fig. 17. For these reasons we will not try to give a reasonable prediction for these decay widths in those corners of the parameter space. From now on we will limit ourselves to point out where they appear, so that the reader is warned that we can not trust the results in those cases. Outside of this dip, there are two different regions. For squark masses larger than $1250 \mathrm{GeV}$ the one-loop correction is around $-45 \%$ whereas the remainder correction (20) is around $-28 \%$ that means that, roughly, one third of the one-loop corrections can be described as coming from the effective couplings (16). Since the corrections (from both: one-loop and effective couplings) are quite large, one can provide the improved (24) description. In the present situation $\delta^{Y u k .-i m p}$ is larger than the corrections from the effective couplings and the fixed-order one-loop corrections, $\delta^{Y u k .-i m p} \sim-57 \%$ but it accounts for two kind of effects: the contribution of higher order terms, and the dependence of the radiative corrections on the external momenta. On the other side of the plot, for squark masses below $1250 \mathrm{GeV}$, the situation is quite different. The one-loop corrections are relatively small (15\%), whereas the effective description gives a slightly smaller result, this region has light particles running in the loops, and the oneloop functions are expected to depend much on the external momenta. In this situation the effective description can not describe properly the radiative corrections. The improved description (24), on the other hand, basically coincides with the fixed order one-loop result. In summary: our improved description includes the higher order terms of the Yukawa- 


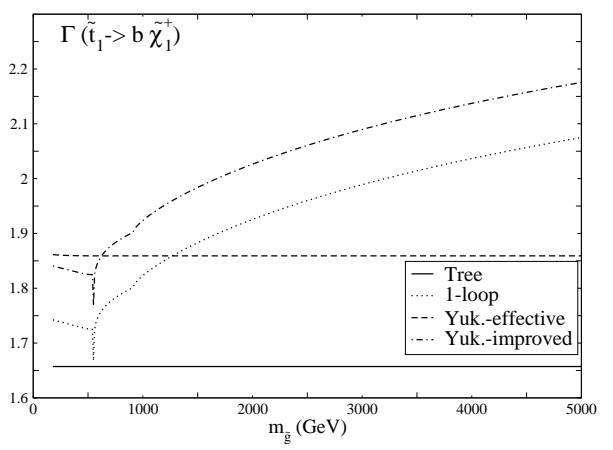

(a)

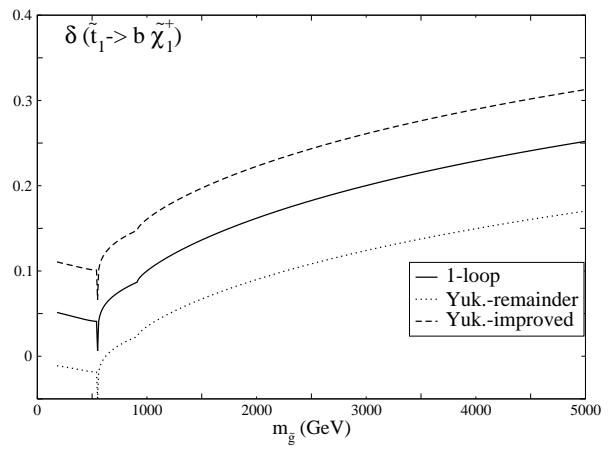

(b)

Figure 2: a) Top-squark partial decay width into the lightest chargino and b) relative corrections, as a function of the gluino mass. Shown are the different approximations: tree-level; one-loop; Yukawa-effective coupling; Yukawa-improved. The input parameters are given in eq. (14).

effective couplings, and the external momenta dependence of the one-loop corrections. It is able to describe both situations: when the one-loop computation gives a sufficient approximation, and when higher order corrections are important and should be taken into account.

Be as it may, it turns out that the effective description using just the Yukawa threshold corrections (16) is not enough for the squark decay widths description. The one-loop corrections develop a term which grows as the gluino mass $m_{\tilde{g}}$ [32], which is absent in the effective Yukawa couplings (16). Fig. 2 shows the comparison of the tree-level, oneloop, and Yukawa-effective computations of the top-squark decay width into charginos, as a function of the gluino mass, for the input parameters of eq. (14). One can clearly see the log-like behaviour of the one-loop corrections, which can not be reproduced using the effective coupling $3^{3}$. The logarithmic terms in the gluino mass are a clear example of the non-decoupling effects. To understand their origin, one can think on the following: in a process where all external (initial and final state) particles belong to the SM sector, one can separate the loop contributions in two kinds: SM-like (with only SM and Higgs boson particles running inside the loops), and non-SM like (with only SUSY particles running inside the loops). Each kind is UV (and IR) finite by itself, so one can remove the nonSM-like part from the computation - or take all non-SM-like particle masses very large without any ill effect. In the present case, however, there are SM and SUSY particles as external states, the Feynman diagrams can no longer be divided into SM-like and non-SM like. The UV divergences from the SM sector are cancelled against UV-divergences of the SUSY-sector, and as a consequence if we remove a SUSY particle from the computation, the computation is UV-divergent - and therefore meaningless. If we try to remove a particle by setting its mass to very large values, this divergence appears as a logarithm of

\footnotetext{
${ }^{3}$ The variation of the Yukawa-effective corrections on the gluino mass (17) in Fig. 2a is of a $0.13 \%$, which can not be appreciated in the plot.
} 
the corresponding particle mass, and we obtain the aforementioned non-decoupling effects. In summary: the QCD corrections to squark decay widths produce explicit non-decoupling terms of the sort $\log m_{\tilde{g}}$.

To include the logarithmic terms in the effective descriptions, we have extracted from the one-loop result the $\log m_{\tilde{g}}$ terms. We have expanded the full one-loop result in the limit $m_{\tilde{g}} \gg m_{\tilde{q}}$, and we have used the reduction factors from Ref. [53] to obtain scalar quantities. The results for top-squark decays into charginos are:

$$
\begin{aligned}
\Delta A_{-a i}^{(t)} & =F_{-a i}^{(t)}+\delta A_{-a i}^{(t)}=-\frac{\alpha_{s}}{2 \pi} A_{-a i}^{(t)} \log \frac{m_{\tilde{g}}^{2}}{\mu_{0}^{2}} \\
\Delta A_{+a i}^{(t)} & =F_{+a i}^{(t)}+\delta A_{+a i}^{(t)}=\left\{\lambda_{t} R_{a 2}^{(t)} V_{i 2}^{*} \frac{\alpha_{s}}{\pi}+\frac{\alpha_{s}}{2 \pi} A_{+a i}^{(t)}\right\} \log \frac{m_{\tilde{g}}^{2}}{\mu_{0}^{2}},
\end{aligned}
$$

here $\Delta A_{+,-}$are the full one-loop corrections to the form factors, $F_{+,-}$are the contributions from the corresponding one-loop diagrams, $\delta A_{+,-}$the counterterm contributions, and $A_{+,-}$are the tree-level couplings defined in (12).$\mu_{0}$ is the scale appearing in the dimensional reduction of the one-loop UV-divergent integrals, which appears when applying the procedure from Ref. [53], and can be though as a renormalization scale. However, these expressions do not give any hint at the origin of the $\log m_{\tilde{g}}$ terms, or how can they be computed. To understand those terms a renormalization group analysis is in order.

\section{Renormalization Group Analysis}

In order to extract the exact dependence on the renormalization scale, we make a renormalization group analysis, which will allow us to compute the logarithmic terms in $m_{\tilde{g}}$. To compute those terms, we construct an effective theory below the gluino mass scale, which contains only squarks, quarks, charginos, neutralinos and gluons in the light sector of the theory, and integrate out the gluino contributions. We find out the renormalization group equations (RGE) of the gaugino and higgsino couplings, and perform the matching with the full MSSM couplings at the gluino mass scale $m_{\tilde{g}}$. In the present computation we will consider only logarithmic RGE effects, and neglect the possible threshold effects at the gluino mass scale. Since the effective theory does not contain gluinos, only the contributions from the gluon have to be taken into account. We will present only the computation for the top-squark decay into charginos, the other couplings follow from the present computation.

\subsection{Gluon contribution to $A_{+a i}^{(t)}$}

The coupling $A_{+a i}^{(t)}$ is the sum of two terms: a gaugino coupling, and a higgsino coupling, as seen in eq. (12). To shorten up the expressions, we will introduce the shortcuts $H_{+}, G$, which represent the higgsino and gaugino part respectively:

$$
A_{+a i}^{(t)}=H_{+}+G \quad ; \quad H_{+}=-\lambda_{t} R_{a 2}^{(t)} V_{i 2}^{*} \quad, \quad G=R_{a 1}^{(t)} V_{i 1}^{*} .
$$


The total gluon contribution to the divergent part of the vertex form factors and the wave function renormalization constants is:

$$
(\text { Coef } \Delta)_{+}=\left(\frac{\alpha_{s}}{3 \pi} A_{+a i}^{(t)}+\frac{1}{2} \frac{\alpha_{s}}{3 \pi} A_{+a i}^{(t)}\right)=\frac{\alpha_{s}}{2 \pi} A_{+a i}^{(t)}
$$

where the first term comes from the vertex form factor's divergent part and the second from the fermion and sfermion wave function renormalization constants. Then, the gluon contribution to the $\beta$ function is

$$
\beta_{+, g}=-2(\operatorname{Coef} \Delta)_{+}=-\frac{\alpha_{s}}{\pi} A_{+a i}^{(t)}=-\frac{\alpha_{s}}{\pi}\left(H_{+}+G\right)
$$

Therefore, the renormalization group equation is

$$
\frac{d A_{+a i}^{(t)}(t)}{d t}=-\frac{\alpha_{s}(t)}{\pi} A_{+a i}^{(t)}(t)
$$

where $t=\log Q, Q$ being the renormalization scale. To solve that equation, we make use of the standard RGE for the QCD coupling constant (see e.g. [54])

$$
\frac{d \alpha_{s}(t)}{d t}=-\frac{1}{2 \pi} \beta_{0} \alpha_{s}^{2}(t)
$$

$\beta_{0}$ being the standard QCD $\beta$-function:

$$
\beta_{0}=\frac{11 N_{c}-2 N_{f}}{3}-\frac{N_{\tilde{f}}}{6}-2 N_{\tilde{g}}
$$

where $N_{c}=3$ is the number of colors, $N_{f}, N_{\tilde{f}}$, and $N_{\tilde{g}}$ are the number of quarks, squarks and gluino that have a mass below the scale $Q$ at which we compute the $\beta_{0}$ function 4 . Inserting expression (30) in (29) we obtain

$$
\frac{d A_{+a i}^{(t)}(t)}{A_{+a i}^{(t)}(t)}=\frac{2}{\beta_{0}} \frac{d \alpha_{s}(t)}{\alpha_{s}(t)}
$$

Solving the equation we obtain

$$
\frac{A_{+a i}^{(t)}(t)}{A_{+a i}^{(t)}\left(t_{0}\right)}=\left(\frac{\alpha_{s}(t)}{\alpha_{s}\left(t_{0}\right)}\right)^{\frac{2}{\beta_{0}}}
$$

Finally, using the QCD running coupling constant

$$
\frac{\alpha_{s}(Q)}{\alpha_{s}\left(Q_{0}\right)}=1-\beta_{0} \frac{\alpha_{s}(Q)}{2 \pi} \log \frac{Q}{Q_{0}}
$$

\footnotetext{
${ }^{4}$ The RGE evolution is performed by steps, taking into account the change in the $\beta_{0}$ function as the scale $Q$ crosses thresholds of colored particles.
} 
the running of the right-handed vertex coupling constant is, approximated to $\mathcal{O}\left(\alpha_{s}\right)$ :

$$
A_{+a i}^{(t)}(Q) \simeq A_{+a i}^{(t)}\left(Q_{0}\right)\left(1-\frac{\alpha_{s}(Q)}{\pi} \log \frac{Q}{Q_{0}}\right) .
$$

The boundary conditions at $Q_{0}=m_{\tilde{g}}$ are

$$
A_{+a i}^{(t)}\left(m_{\tilde{g}}\right)=H_{+}\left(m_{q}\left(m_{\tilde{g}}\right)\right)+G\left(m_{\tilde{g}}\right),
$$

then, the running coupling constant is

$$
A_{+a i}^{(t)}(Q) \simeq\left(H_{+}\left(m_{q}\left(m_{\tilde{g}}\right)\right)+G\left(m_{\tilde{g}}\right)\right)\left(1-\frac{\alpha_{s}(Q)}{\pi} \log \frac{Q}{m_{\tilde{g}}}\right) .
$$

Now, we have the squark-chargino running coupling constant as a function of the gauge and Higgs boson couplings at the gluino mass scale, but we want to express it as a function of couplings at the renormalization scale $Q$. Note that the gauge part of the coupling $G$ (26) only contains EW gauge couplings, and they do not receive one-loop running contributions from the QCD sector, therefore it is a constant term.

$$
G\left(m_{\tilde{g}}\right)=G(Q) \equiv G \text {. }
$$

The higgsino coupling $H_{+}(26)$, on the other hand, has a dependence on the quark Yukawa coupling (or mass), which does run due to QCD corrections, according to the RGE,

$$
m(Q)=m\left(Q_{0}\right)\left(\frac{\alpha_{s}(Q)}{\alpha_{s}\left(Q_{0}\right)}\right)^{\frac{4}{\beta_{0}}}
$$

by inserting these expression into eq. (32) we can obtain

$$
\begin{aligned}
A_{+a i}^{(t)}(Q) & =A_{+a i}^{(t)}\left(m_{\tilde{g}}\right)\left(\frac{\alpha_{s}(Q)}{\alpha_{s}\left(m_{\tilde{g}}\right)}\right)^{\frac{2}{\beta_{0}}} \\
& =H_{+}\left(m_{q}\left(m_{\tilde{g}}\right)\right)\left(\frac{\alpha_{s}(Q)}{\alpha_{s}\left(m_{\tilde{g}}\right)}\right)^{\frac{2}{\beta_{0}}}+G\left(\frac{\alpha_{s}(Q)}{\alpha_{s}\left(m_{\tilde{g}}\right)}\right)^{\frac{2}{\beta_{0}}} \\
& =H_{+}\left(m_{q}(Q)\right)\left(\frac{\alpha_{s}(Q)}{\alpha_{s}\left(m_{\tilde{g}}\right)}\right)^{\frac{-2}{\beta_{0}}}+G\left(\frac{\alpha_{s}(Q)}{\alpha_{s}\left(m_{\tilde{g}}\right)}\right)^{\frac{2}{\beta_{0}}} \\
& \simeq H_{+}\left(m_{q}(Q)\right)\left(1+\frac{\alpha_{s}(Q)}{\pi} \log \frac{Q}{m_{\tilde{g}}}\right)+G\left(1-\frac{\alpha_{s}(Q)}{\pi} \log \frac{Q}{m_{\tilde{g}}}\right)
\end{aligned}
$$

where, in the last line, we have made the $\mathcal{O}\left(\alpha_{s}\right)$ approximation. Note that the expressions for the higgsino and gaugino couplings are different. Actually, if we write the higgsino and gaugino couplings at the scale $Q$ as a function of the couplings at the scale $m_{\tilde{g}}$ they have the same form (36), since they have the same RGE (32). The difference appears when we write the higgsino/gaugino couplings at the scale $Q$ as a function of the gauge/Higgs couplings at the same scale (39), due to the different running of the gauge (37) and Higgsboson (38) couplings between the scales $m_{\tilde{g}}$ and $Q$. The last line in eq. (39) agrees with the $\log m_{\tilde{g}} / \mu_{0}$ term of the fixed order one-loop expression in (25). 


\subsection{Gluon contribution to $A_{-a i}^{(t)}$}

The gluon contribution to the divergent part of the left-handed couplings $A_{-a i}^{(t)}$, eq. (12), is the same that in the previous case.

$$
(\text { Coef } \Delta)_{-}=\left(\frac{\alpha_{s}}{3 \pi} A_{-a i}^{(t)}+\frac{1}{2} \frac{\alpha_{s}}{3 \pi} A_{-a i}^{(t)}\right)=\frac{\alpha_{s}}{2 \pi} A_{-a i}^{(t)},
$$

But now, the coupling only contains a Yukawa like coupling

$$
\beta_{-, g}=-2(\operatorname{Coef} \Delta)_{-}=-\frac{\alpha_{s}}{\pi} A_{-a i}^{(t)}=-\frac{\alpha_{s}}{\pi} H_{-} .
$$

The renormalization group equation is

$$
\frac{d H_{-}(t)}{d t}=-\frac{\alpha_{s}(t)}{\pi} H_{-}(t)
$$

which has as a solution

$$
H_{-}(Q)=H_{-}\left(m_{q}\left(m_{\tilde{g}}\right)\right)\left(\frac{\alpha_{s}(Q)}{\alpha_{s}\left(m_{\tilde{g}}\right)}\right)^{\frac{2}{\beta_{0}}} .
$$

Following the same steps as in the the previous section, we obtain

$$
\begin{aligned}
H_{-}(Q) & =H_{-}\left(m_{q}\left(m_{\tilde{g}}\right)\right)\left(\frac{\alpha_{s}(Q)}{\alpha_{s}\left(m_{\tilde{g}}\right)}\right)^{\frac{2}{\beta_{0}}}=H_{-}\left(m_{q}(Q)\right)\left(\frac{\alpha_{s}(Q)}{\alpha_{s}\left(m_{\tilde{g}}\right)}\right)^{\frac{-2}{\beta_{0}}} \\
& \simeq H_{-}\left(m_{q}(Q)\right)\left(1+\frac{\alpha_{s}(Q)}{\pi} \log \frac{Q}{m_{\tilde{g}}}\right)
\end{aligned}
$$

where in the last line we have made the $\mathcal{O}\left(\alpha_{s}\right)$ approximation. This expression coincides with the higgsino running coupling constant for $A_{+a i}^{(t)}$ (39), and it also agrees with the $\log m_{\tilde{g}} / \mu_{0}$ term of the fixed order one-loop expression in (25).

\subsection{Renormalization Group summary}

The renormalization group running of the coupling constant, can be summarized as follows: we can use effective gaugino and higgsino couplings given by,

$$
\begin{aligned}
& g^{e f f \cdot(Q)}=g\left(\frac{\alpha_{s}(Q)}{\alpha_{s}\left(m_{\tilde{g}}\right)}\right)^{\frac{2}{\beta_{0}}} \simeq g\left(1-\frac{\alpha_{s}(Q)}{\pi} \log \frac{Q}{m_{\tilde{g}}}\right), \\
& \tilde{\lambda}_{b, t}^{e f f \cdot}(Q)=\lambda_{b, t}^{e f f \cdot}(Q)\left(\frac{\alpha_{s}(Q)}{\alpha_{s}\left(m_{\tilde{g}}\right)}\right)^{\frac{-2}{\beta_{0}}} \simeq \lambda_{b, t}^{e f f \cdot}(Q)\left(1+\frac{\alpha_{s}(Q)}{\pi} \log \frac{Q}{m_{\tilde{g}}}\right),
\end{aligned}
$$

where $\lambda^{\text {eff. }}(Q)$ are the effective Yukawa couplings defined in (16). We define then an effective partial decay width, computed by replacing $g$ and $\lambda$ in the tree-level expression (13) by the expressions of eq. (45):

$$
\Gamma^{e f f .}=\Gamma^{\text {tree }}\left(g^{e f f .}, \tilde{\lambda}^{e f f \cdot}(Q)\right)
$$




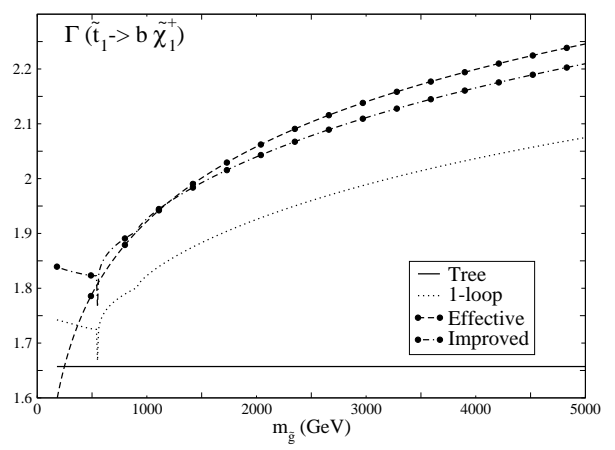

(a)

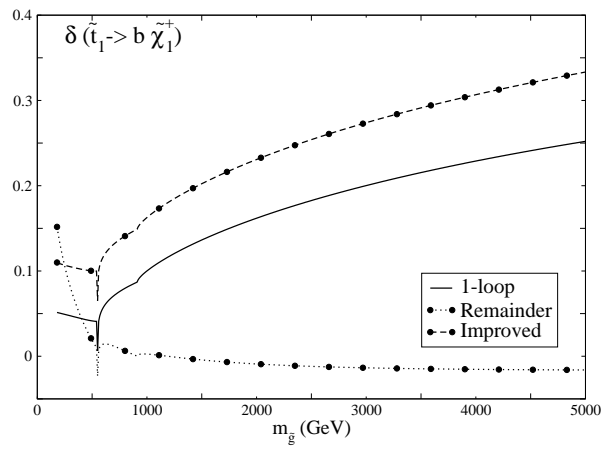

(b)

Figure 3: a) Partial decay widths and b) relative corrections of the top-squark decay into the lightest chargino, as a function of the gluino mass $m_{\tilde{g}}$. Shown are different approximations to the computation. The effective, improved and remainder computations include the $\log m_{\tilde{g}} / Q$ terms. The input parameters are given in eq. (14).

and we define improved and remainder widths and corrections ( $\left.\Gamma^{i m p .}, \delta^{i m p .}, \delta^{r e m .}\right)$, in the same fashion as the Yukawa-effective, Yukawa-improved and Yukawa-remainder of eqs. (19), (20), (24).

The origin of the $\log m_{\tilde{g}}$ terms is a consequence of the SUSY-breaking, and, technically, can be seen in different ways, depending on the approximation used to make the computation. In the one-loop fixed order computation they appear because of the cancellation of UV-divergences between the gluon and gluino loops (as explained above), but in the effective theory point of view, they appear because of the different running of the gauge/Higgs boson and gaugino/higgsino couplings. In a fully SUSY theory the gaugino (higgsino) couplings are equal to the gauge (Higgs) boson couplings, and they have the same RGE, but in a theory with broken SUSY (as the present one) these couplings are no longer the same, they have different RGE, and the difference between them is a measure of the SUSY-breaking $\left(\log m_{\tilde{g}}\right)$.

Fig. 3 shows the partial decay width of the top-squark into the lightest chargino, where we include the $\log m_{\tilde{g}}$ terms of eq. (45) in the effective description. The input parameters are given in eq. (14). Now the effective description follows the logarithmic behaviour of the full one-loop corrections. Moreover, we have checked the validity of our result by comparing the full one-loop corrections in the limit $m_{\tilde{g}} \gg M$ with the one-loop expansion of the effective description, which contains only $\log m_{\tilde{g}} / \mu_{0}$ terms. The results agree with the previous results of Ref. [32,34] in the limit $m_{\tilde{g}} \gg m_{\tilde{q}}$. Our results go beyond the ones of Ref. [32], by including the Yukawa terms, including explicitly all the chargino/neutralino and squark mixing and couplings, showing the exact dependence from the renormalization group, and performing (see below) the numerical comparison with the fixed order computation.

Fig. 3b shows the new value of the remainder contributions. At low values of the gluino

\footnotetext{
${ }^{5}$ The comparison is performed by comparing the slope of the different computations in the plots as a function of $\log \frac{m_{\tilde{g}}}{\mu_{0}}$.
} 
mass, the remainder is still large, because $m_{\tilde{g}} \simeq m_{\tilde{q}}$ and the logarithmic approximation does not make sense, but for $m_{\tilde{g}} \geq 1 \mathrm{TeV}$ the remainder contributions stay below the $2 \%$ level.

\section{$5 \quad$ Numerical analysis}

Following the computation and setup of the previous sections, we perform a complete numerical analysis. We will concentrate on third generation squark decays (top-squarks and bottom-squarks), as they have the richest phenomenology, since only on them the higgsino couplings are large enough. The input parameters that we use are given in eq. (14), and the resulting spectrum is discussed in section 2.1. We have chosen a large value for the gluino mass to enhance the effects of the logarithmic terms.

As a first example we show in Fig. [4 the relative corrections to the squark (stop and sbottom) partial decay widths into charginos and neutralinos, as a function of the gluino mass $\left(m_{\tilde{g}}\right)$. We show the predictions of the effective description including only the effects of the effective Yukawa couplings (16) (labelled Yuk.), and including also the $\log m_{\tilde{g}} / Q$ terms of eq. (45) (lines marked with full circles). We show the partial decays into the two charginos and two neutralinos $\chi_{1}^{0}, \chi_{3}^{0}$, the results for the other neutralinos are similar to the ones shown. The second neutralino $\left(\chi_{2}^{0}\right)$ is mostly a gaugino $(\tilde{w})$ and its results are very similar to $\chi_{1}^{-}$, whereas the fourth neutralino $\left(\chi_{4}^{0}\right)$ is mostly of higgsino-type $(\tilde{h})$ and its results are very similar to $\chi_{3}^{0}$. In all plots we observe the same pattern: the effective Yukawa couplings (16) do not describe correctly the variation with $m_{\tilde{g}}$, only after including the log-terms of eq. (45) does the effective description follow the shape of the one-loop corrections. The remainder corrections $\delta^{Y u k .-r e m}$. (20) - those terms that are not described by the effective couplings - have a dependence on $m_{\tilde{g}}$ before including the $\log$-terms, but after including them we see that in all channels $\delta^{r e m}$. is essentially flat above $m_{\tilde{g}} \sim 1500 \mathrm{GeV}$, which means that they have absorbed the bulk of the dependence on $m_{\tilde{g}}$. Moreover, after including the log-terms $\delta^{r e m}$. is much smaller - between a $-2 \%$ and a $-5 \%$ - than without them - between a $5 \%$ and a $-10 \%$. The effects of the log-terms are more visible in the gaugino-like channels, where the Yukawa couplings play no role, and the bulk of the corrections corresponds to the log-terms. In these channels the corrections change in a range of a $10 \%$ in the interval $m_{\tilde{g}}=[500,5000] \mathrm{GeV}$. In the higgsino-like channels their importance is less apparent. On one side the effective Yukawa couplings carry the bulk of the corrections (around a $-30 \%$ ), and on the other side the sign difference among the two contributions (45) make them to partially compensate each other. But also in this case the effective Yukawa couplings alone (16) do not describe correctly the $m_{\tilde{g}}$-dependence, and there is a range of a $3 \%$ variation in the corrections in the studied $m_{\tilde{g}}$-interval. The spikes that are seen in some plots correspond to the threshold singularities for the opening of the squark decay into gluinos $(\tilde{q} \rightarrow \tilde{g} q)$, we recall that in this work we are not interested in the region where the gluino decay channel is open $\left(m_{\tilde{g}}+m_{q}<m_{\tilde{q}}\right)$, since in that region the strong decay is the leading one, and the chargino/neutralino channels have a negligible branching ratio, and are phenomenologically irrelevant. Nevertheless we include the plots 

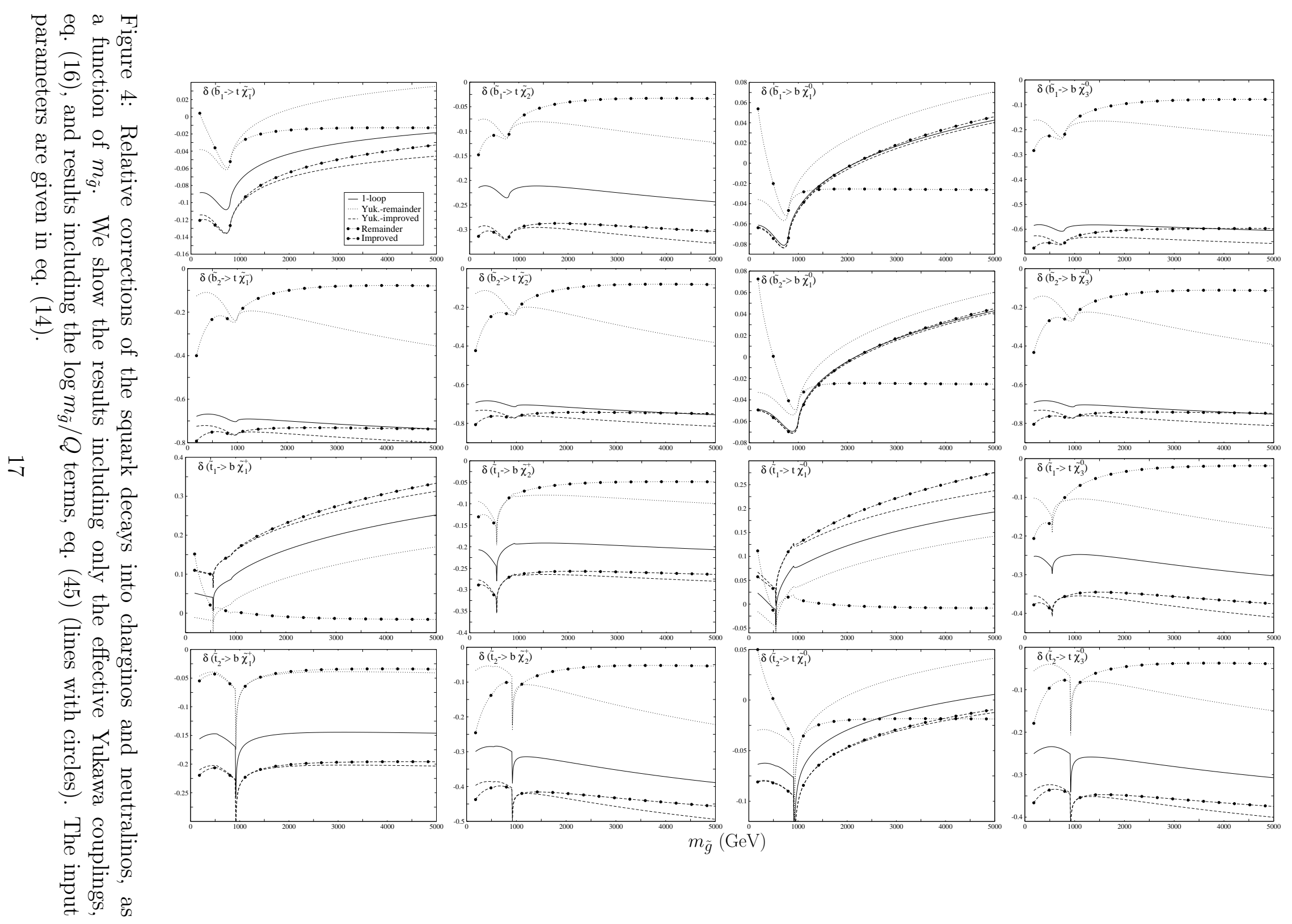

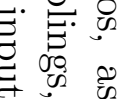




\begin{tabular}{||c|c|c||c|c|c||}
\hline Channel & $\delta^{\text {Yuk.-rem. }}$ & $\delta^{\text {rem. }}$ & Channel & $\delta^{\text {Yuk.-rem. }}$ & $\delta^{r e m .}$ \\
\hline$\tilde{b}_{1} \rightarrow t \chi_{1}^{-}$ & $2.4 \%$ & $-1.5 \%$ & $\tilde{t}_{1} \rightarrow t \chi_{1}^{+}$ & $-10 \%$ & $-6 \%$ \\
\hline$\tilde{b}_{1} \rightarrow t \chi_{2}^{-}$ & $-10 \%$ & $-3 \%$ & $\tilde{t}_{1} \rightarrow t \chi_{2}^{+}$ & $-29 \%$ & $-12 \%$ \\
\hline$\tilde{b}_{1} \rightarrow b \chi_{1}^{0}$ & $-18 \%$ & $-8 \%$ & $\tilde{t}_{1} \rightarrow t \chi_{1}^{0}$ & $10 \%$ & $-0.6 \%$ \\
\hline$\tilde{b}_{1} \rightarrow b \chi_{3}^{0}$ & $-51 \%$ & $-18 \%$ & $\tilde{t}_{1} \rightarrow t \chi_{3}^{0}$ & $-14 \%$ & $-2 \%$ \\
\hline$\tilde{b}_{2} \rightarrow t \chi_{1}^{-}$ & $-67 \%$ & $-19 \%$ & $\tilde{t}_{2} \rightarrow t \chi_{1}^{+}$ & $-6 \%$ & $-4 \%$ \\
\hline$\tilde{b}_{2} \rightarrow t \chi_{2}^{-}$ & $-31 \%$ & $-10 \%$ & $\tilde{t}_{2} \rightarrow t \chi_{2}^{+}$ & $-19 \%$ & $-7 \%$ \\
\hline$\tilde{b}_{2} \rightarrow b \chi_{1}^{0}$ & $-12 \%$ & $-6 \%$ & $\tilde{t}_{2} \rightarrow t \chi_{1}^{0}$ & $3 \%$ & $-1.8 \%$ \\
\hline$\tilde{b}_{2} \rightarrow b \chi_{3}^{0}$ & $-40 \%$ & $-16 \%$ & $\tilde{t}_{2} \rightarrow t \chi_{3}^{0}$ & $-11 \%$ & $-4 \%$ \\
\hline \hline
\end{tabular}

Table 1: Value of the remainder corrections (20) for specific squark partial decay widths, including only the effective Yukawa couplings, eq. (16), and results including the $\log m_{\tilde{g}} / Q$ terms, eq. (45), for $\tan \beta=50$, and the rest of input parameters as given in eq. (14).

also in that region to show the trend of the corrections.

Next, we show in Fig. 5 the evolution of the different corrections as a function of $\tan \beta$. Here we see large negative corrections, growing with $\tan \beta$. The origin of the negative corrections is twofold: on one side the standard QCD running of the quark mass reduces significantly the Yukawa coupling, and on the other, for positive values of the higgsino mass parameter $\mu$ the contributions to $\Delta m_{q}$ (17) are also positive, decreasing even more the effective Yukawa couplings (16). However, even after taking into account these two sources of corrections, still there is a large remainder of relative corrections (up to $-67 \%$ at $\tan \beta=50$ for some channels) which can not be accounted for. After including the logarithmic terms, eq. (45), the situation is quite different. Now, the effective description can reproduce quite well the one-loop results, and the remainder corrections (those that can not be described by the effective couplings) are reduced at a level (in absolute value) below the 20\%. Table 1 shows the value of the remainder corrections (20) for all studied channels at $\tan \beta=50$, including the full effective description, and including only the Yukawa corrections. We see that in all channels the corrections are reduced significantly after including the logarithmic terms.

Finally Fig. 6 shows the evolution of the corrections to the partial decay widths as a function of the $S U(2)_{L}$ squark mass parameter $M_{\tilde{f}_{\mathrm{L}}}$. The abrupt change which is seen at the middle of the plots corresponds with the situation in which $M_{\tilde{f}_{\mathrm{L}}} \simeq M_{\tilde{f}_{\mathrm{R}}}$, and the physical states suffer an abrupt change between left and right chirality. This explains the difference in value and behaviour of the corrections in the regions of $M_{\tilde{f}_{\mathrm{L}}}$ below and above that point. This is also the situation, shown in Fig. 1, where the partial decay widths can become zero, and the one-loop corrections can become non-perturbative. In all situations the description including the log-terms provides a better description of the radiative corrections with a $\delta^{r e m}$ (20) much smaller than with the effective Yukawa couplings (16) alone, and a much softer variation, meaning that the description of eq. (45) is accurate for all values of the squark mass. Let us remember, that by changing the SUSY parameter $M_{\tilde{f}_{\mathrm{L}}}$, the physical squark masses also change, and that since the renormalization scale is taken to 


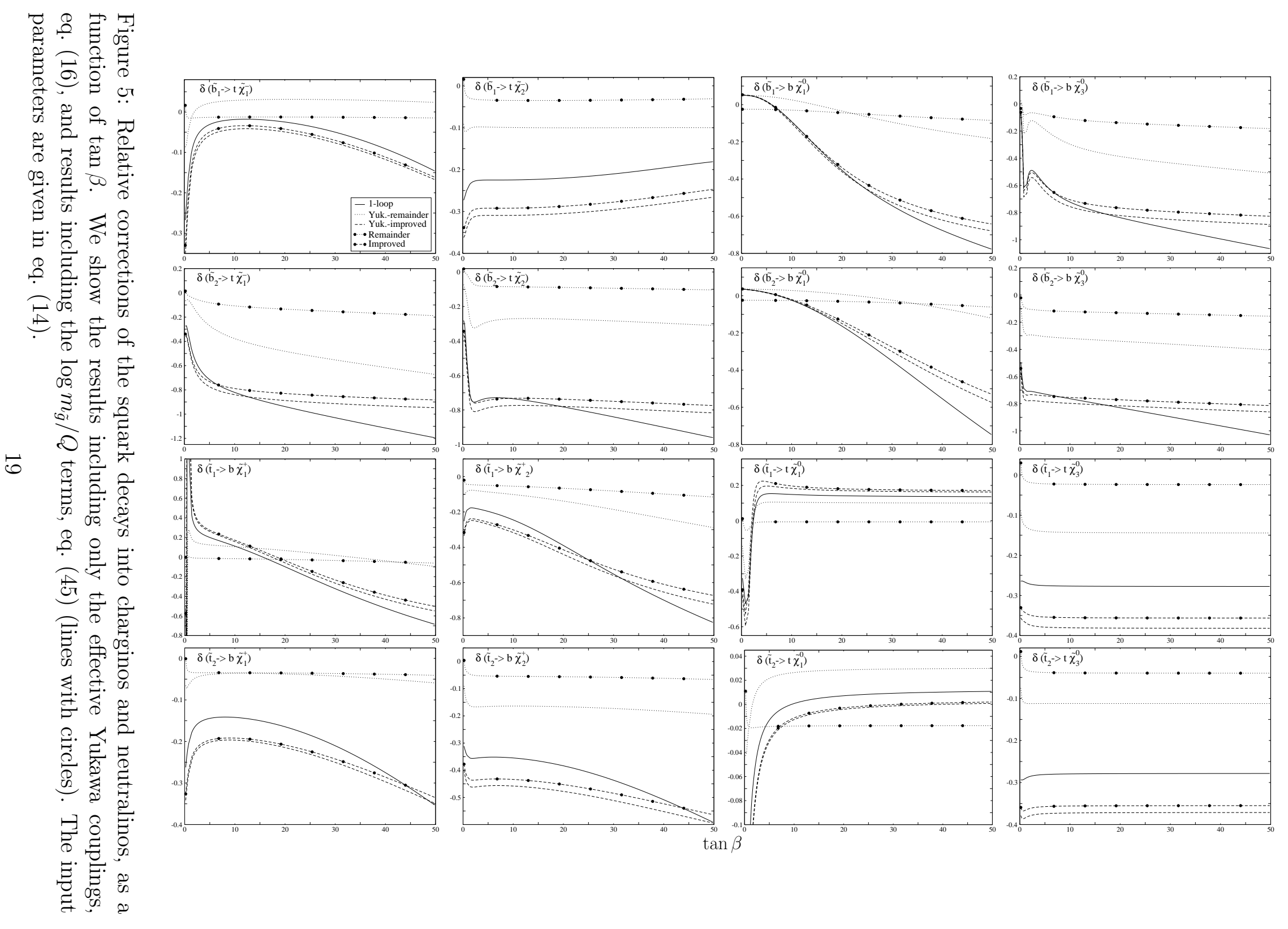



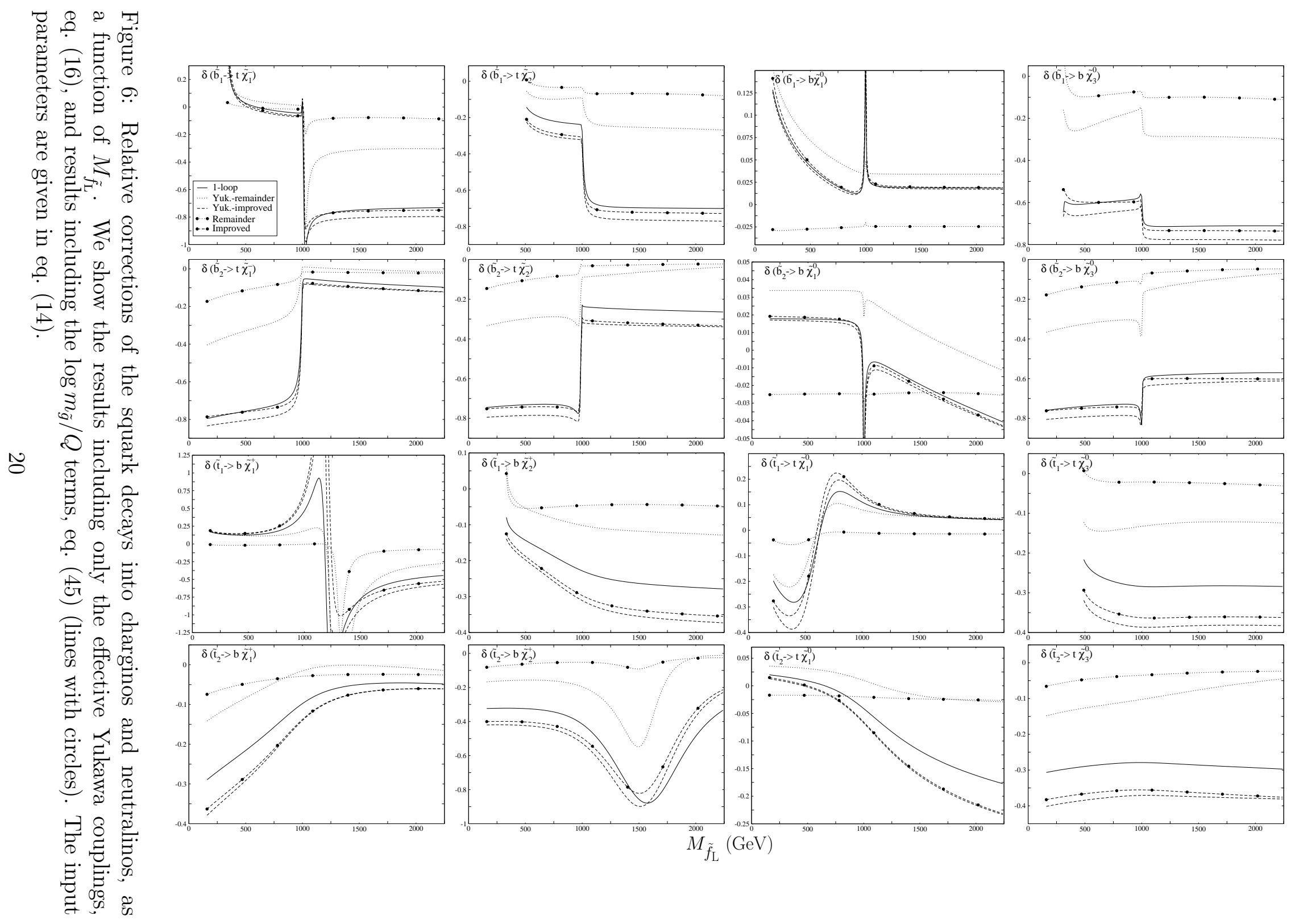

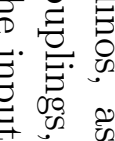

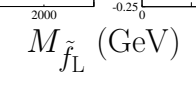


be the decaying squark mass, the log-terms of eq. (45) effectively run also with $M_{\tilde{f}_{\mathrm{L}}}$ as $\sim \log m_{\tilde{g}} / M_{\tilde{f_{\mathrm{L}}}}$ (for mostly-left-handed squarks) - a contribution that can not be described with the effective Yukawa couplings (16).

\section{$6 \quad$ Summary and conclusions}

We have proposed and analyzed an effective description of squark interactions with charginos and neutralinos in the MSSM. We have applied it to the partial decay widths of squarks into charginos and neutralinos. We have compared it with the full one-loop corrections, and have proposed a way to combine the effective description (which includes higher order terms) with the complete one-loop description (which includes all kinetic and mass-effects factors), providing an improved computation, $\Gamma^{i m p}$. (19). The difference between the effective description and the improved computation is encoded in the remainder contribution,

$\delta^{r e m}$. eq. (20), which gives a measure of the precision of the effective description.

The effective description includes the effective Yukawa couplings (16), which take into account the resummation effects [49-52]. Note that the computation of the threshold corrections (17) includes only the $(\tan \beta, \cot \beta)$ proportional terms, since the terms that would be proportional to the trilinear couplings $\left(A_{b}, A_{t}\right)$ are actually subleading [52]. This description produces large remainder $\delta^{Y u k .-r e m}$. corrections, and does not reproduce the behaviour of the one-loop corrections as a function of several parameters - notably it is missing a $\log m_{\tilde{g}}$ term. Therefore, we conclude that it does not reproduce satisfactorily the one-loop corrections, and it is not a good approximation.

We have computed the missing $\log m_{\tilde{g}}$ terms with the help of the renormalization group - eq. (45) -, and found agreement between the renormalization group analysis and the large mass expansion of the one-loop result (25). After including also the $\log m_{\tilde{g}}$-terms of eq. (45), the effective description produces a reasonable approximation to the radiativecorrected partial decay widths of squarks into charginos and neutralinos, as shown by a small absolute value of the remainder contributions $\delta^{r e m}$, and by a nearly-flat behaviour of the corrections as a function of different parameters.

The origin of the logarithmic terms can be explained in different (complementary) ways, depending on the kind of approximation that we take. First of all, from the fundamental point of view, they are non-decoupling terms that appear due to the supersymmetrybreaking. Since we are testing SUSY relations (equality of the gauge/Yukawa couplings to the gaugino/higgsino couplings), and SUSY is broken (by the term $m_{\tilde{g}}$ among others), we have to find some effect that tells us about the breaking of SUSY at that scale - e.g. a $\log m_{\tilde{g}}$-term. Second, from a fixed-order one-loop description point of view, the SUSY relation appears because the UV-divergences of the loops containing gluinos cancel with the UV-divergences of the loops containing gluons, and the log-terms that accompany those loops combine between them - producing a $\log m_{\tilde{g}^{-}}$term. And third, from a renormalization group (and effective theory) point of view, the gaugino/higgsino couplings run different than the gauge/Yukawa couplings in the region where SUSY is broken, that is, for scales below $m_{\tilde{g}}$. The running of the gaugino/higgsino couplings from the scale $m_{\tilde{g}}$ to 
the chosen renormalization scale produces $\log m_{\tilde{g}} / Q$-terms. Since the Yukawa couplings already include some QCD running, whereas the EW-gauge couplings do not, the relation between the gauge/gaugino and Yukawa/higgsino couplings has some differences (45).

The presence of the non-decoupling $\log m_{\tilde{g}}$-terms implies a deviation of the equality between the higgsino/gaugino and Higgs/gauge couplings predicted by exact SUSY. This deviation is important, and has to be taken into account in the experimental measurement of SUSY relations. At the same time, it gives us access to information about heavy particles that can not be directly produced at the LHC/ILC. For these reasons it is important to include these effects in the computation for the predictions of squark observables at the LHC and the ILC. The effective description of squark/chargino/neutralino couplings given by eqs. (16), (17), (45), is simple to write, and to introduce in computer codes, it costs little computational power, and provides a reasonable description for squark decays into charginos and neutralinos, so it can be used in monte-carlo generators and other computer programs that provide predictions for the LHC and the ILC to improve their accuracy at a minimum cost.

\section{Acknowledgements}

J.G. and R.S.F. have been supported in part by MEC and FEDER under project FPA200766665C02-02, J.G. also by DURSI Generalitat de Catalunya under project 2005SGR00564; S.P. by a Ramón y Cajal contract from MEC (Spain) (PDRYC-2006-000930) and partially by CICYT (FPA2006-2315) and DGIID-DGA (2008-E24/2); R.S.F. also by a MEC FPI grant (BES-2005-8861). This work has also been supported by the Spanish ConsoliderIngenio 2010 Programme CPAN (CSD2007-00042). J.G. wishes to thank the hospitality of the Universidad de Zaragoza, and S.P. wishes to thank the hospitality of the Universitat de Barcelona, where part of this work was done.

\section{References}

[1] C. Amsler et al. (Particle Data Group Collaboration), Phys. Lett. B667, 1 (2008).

[2] H. E. Haber, arXiv:hep-ph/9308209, Proceedings of Recent advances in the superworld,Woodlands, USA, 13-16 Apr 1993, pp. 37-51, eds. J.L. Lopez and D.V. Nanopoulos.

[3] ATLAS Collaboration, CERN-LHCC-99-14, CERN-LHCC-99-15, ATLAS: Detector and physics performance technical design report. Volumes 1,2 .

[4] G. L. Bayatian et al. (CMS Collaboration), J. Phys. G34, 995-1579 (2007).

[5] J. A. Aguilar-Saavedra et al. (ECFA/DESY LC Physics Working Group Collaboration), arXiv:hep-ph/0106315, TESLA Technical Design Report Part III: Physics at an $e^{+} e^{-}$Linear Collider, R. Heuer, D.J. Miller, F. Richard, P.M. Zerwas Editors. 
[6] G. Weiglein et al. (LHC/LC Study Group Collaboration), Phys. Rept. 426, 47-358 (2006), arXiv:hep-ph/0410364.

[7] H. P. Nilles, Phys. Rept. 110, 1 (1984).

[8] H. E. Haber and G. L. Kane, Phys. Rept. 117, 75 (1985).

[9] A. B. Lahanas and D. V. Nanopoulos, Phys. Rept. 145, 1 (1987).

[10] S. Ferrara, editor, Supersymmetry, volume 1-2, North Holland/World Scientific, Singapore, 1987.

[11] P. de Jong, arXiv:0809.3708 [hep-ex], to appear in the proceedings of The 16th International Conference on Supersymmetry and the Unification of Fundamental Interactions (SUSY08), June 16 - 21, 2008, Seoul, Korea.

[12] J. Ellis, arXiv:0810.1178 [hep-ph].

[13] J. L. Feng, M. E. Peskin, H. Murayama and X. R. Tata, Phys. Rev. D52, 1418-1432 (1995), arXiv:hep-ph/9502260.

[14] H.-C. Cheng, J. L. Feng and N. Polonsky, Phys. Rev. D57, 152-169 (1998), arXiv:hep-ph/9706476.

[15] A. Freitas et al., Nucl. Phys. B - Proc. Suppl. 117, Supplement 1, 807-810 (2003), arXiv:hep-ph/0211108, Proceedings of 31st International Conference on High Energy Physics (ICHEP 2002), Amsterdam, The Netherlands, 24-31 Jul 2002, p.807 and proceedings of International Workshop on Linear Colliders (LCWS 2002), Jeju Island, Korea, 26-30 Aug 2002, p.189.

[16] A. Bartl, W. Majerotto and W. Porod, Z. Phys. C64, 499-508 (1994), Erratum ibid C68, 518 (1995).

[17] W. Beenakker, R. Hopker and P. M. Zerwas, Phys. Lett. B378, 159-166 (1996), arXiv:hep-ph/9602378.

[18] W. Beenakker, R. Hopker, T. Plehn and P. M. Zerwas, Z. Phys. C75, 349-356 (1997), arXiv:hep-ph/9610313.

[19] A. Bartl et al., Phys. Lett. B419, 243-252 (1998), arXiv:hep-ph/9710286.

[20] A. Bartl et al., Phys. Lett. B435, 118-124 (1998), arXiv:hep-ph/9804265.

[21] A. Bartl et al., Phys. Rev. D59, 115007 (1999), arXiv:hep-ph/9806299.

[22] A. Bartl et al., Phys. Lett. B460, 157-163 (1999), arXiv:hep-ph/9904417.

[23] K.-i. Hikasa and M. Kobayashi, Phys. Rev. D36, 724 (1987). 
[24] T. Han, K.-i. Hikasa, J. M. Yang and X.-m. Zhang, Phys. Rev. D70, 055001 (2004), arXiv:hep-ph/0312129.

[25] F. del Aguila et al., Eur. Phys. J. C57, 183-308 (2008), arXiv:0801.1800 [hep-ph], Report of Working Group 1 of the CERN Workshop Flavor in the Era of the LHC: A Workshop on the Interplay of Flavor and Collider Physics, Geneva, Switzerland, November 2005 - March 2007.

[26] W. Porod and T. Wohrmann, Phys. Rev. D55, 2907-2917 (1997), arXiv:hep-ph/9608472, Erratum ibid D67, 059902 (2003).

[27] W. Porod, Phys. Rev. D59, 095009 (1999), arXiv:hep-ph/9812230.

[28] C. Boehm, A. Djouadi and Y. Mambrini, Phys. Rev. D61, 095006 (2000), arXiv:hep-ph/9907428.

[29] A. Djouadi and Y. Mambrini, Phys. Lett. B493, 120-126 (2000), arXiv:hep-ph/0007174.

[30] S. P. Das, A. Datta and M. Guchait, Phys. Rev. D65, 095006 (2002), arXiv:hep-ph/0112182.

[31] A. Djouadi and Y. Mambrini, Phys. Rev. D63, 115005 (2001), arXiv:hep-ph/0011364.

[32] K.-i. Hikasa and Y. Nakamura, Z. Phys. C70, 139-144 (1996), arXiv:hep-ph/9501382.

[33] S. Kraml, H. Eberl, A. Bartl, W. Majerotto and W. Porod, Phys. Lett. B386, 175-182 (1996), arXiv:hep-ph/9605412.

[34] A. Djouadi, W. Hollik and C. Junger, Phys. Rev. D55, 6975-6985 (1997), arXiv:hep-ph/9609419.

[35] J. Guasch, W. Hollik and J. Solà, Phys. Lett. B437, 88-99 (1998), arXiv: hep-ph/9802329.

[36] J. Guasch, W. Hollik and J. Solà, Phys. Lett. B510, 211-220 (2001), arXiv:hep-ph/0101086.

[37] J. Guasch, W. Hollik and J. Solà, JHEP 10, 040 (2002), arXiv:hep-ph/0207364.

[38] J. Guasch, W. Hollik and J. Solà, Nucl. Phys. B - Proc. Suppl. 116, 301 (2003), arXiv:hep-ph/0210118.

[39] T. Hahn and C. Schappacher, Comput. Phys. Commun. 143, 54-68 (2002), arXiv:hep-ph/0105349. 
[40] J. M. Frere, D. R. T. Jones and S. Raby, Nucl. Phys. B222, 11 (1983).

[41] M. Claudson, L. J. Hall and I. Hinchliffe, Nucl. Phys. B228, 501 (1983).

[42] C. Kounnas, A. B. Lahanas, D. V. Nanopoulos and M. Quiros, Nucl. Phys. B236, 438 (1984).

[43] J. F. Gunion, H. E. Haber and M. Sher, Nucl. Phys. B306, 1 (1988).

[44] J. Küblbeck, M. Böhm and A. Denner, Comput. Phys. Commun. 60, 165-180 (1990).

[45] G. J. van Oldenborgh and J. A. M. Vermaseren, Z. Phys. C46, 425-438 (1990).

[46] T. Hahn and M. Pérez-Victoria, Comput. Phys. Commun. 118, 153 (1999), arXiv:hep-ph/9807565.

[47] T. Hahn, Comput. Phys. Commun. 140, 418-431 (2001), arXiv:hep-ph/0012260.

[48] T. Hahn, FeynArts, FormCalc and LoopTools user's guides, available from http://www.feynarts.de.

[49] J. A. Coarasa, D. Garcia, J. Guasch, R. A. Jiménez and J. Solà, Eur. Phys. J. C2, 373-392 (1998), arXiv:hep-ph/9607485.

[50] M. Carena, D. Garcia, U. Nierste and C. E. M. Wagner, Nucl. Phys. B577, 88-120 (2000), arXiv:hep-ph/9912516.

[51] J. Guasch, W. Hollik and S. Peñaranda, Phys. Lett. B515, 367-374 (2001), arXiv:hep-ph/0106027.

[52] J. Guasch, P. Häfliger and M. Spira, Phys. Rev. D68, 115001 (2003), arXiv:hep-ph/0305101.

[53] G. Devaraj and R. G. Stuart, Nucl. Phys. B519, 483-513 (1998), arXiv: hep-ph/9704308.

[54] A. J. Buras, arXiv:hep-ph/9806471, in "Probing the Standard Model of Particle Interactions" (Les Houches lectures session LXVIII), R. Gupta, A. Morel, E. de Rafael, F. David, Eds., North Holland (1999). 